\title{
Somatostatin prevents lipopolysaccharide-induced neurodegeneration in the rat substantia nigra by inhibiting the activation of microglia
}

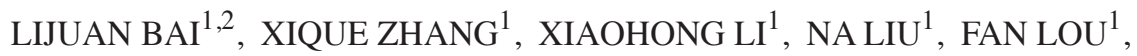 \\ HONGLEI MA ${ }^{1}$, XIAOGUANG LUO ${ }^{1}$ and YAN REN ${ }^{1}$ \\ ${ }^{1}$ Department of Neurology, The First Affiliated Hospital of China Medical University, Shenyang, Liaoning 110001; \\ ${ }^{2}$ Department of Neurology, The People's Hospital of Liaoning Province, Shenyang, Liaoning 110016, P.R. China
}

Received May 21, 2014; Accepted January 30, 2015

DOI: $10.3892 / \mathrm{mmr} .2015 .3494$

\begin{abstract}
Somatostatin (SST) is a neuromodulator which is abundant throughout the central nervous system (CNS) and has a crucial role in neurodegenerative disorders. However, little is known about the effects and mechanisms of SST in dopaminergic (DA) neurons in the context of Parkinson's disease (PD). In the present study, a model of PD was generated by injecting lipopolysaccharide (LPS) into the substantia nigra (SN) of rats in order to investigate the effects of SST on LPS-induced degeneration of DA in vivo. Intramural injection of LPS resulted in a significant loss of DA neurons, while reduction of neuronal death by SST pretreatment was confirmed using immunohistochemical staining for tyrosine hydroxylase and Nissl. In parallel, immunohistochemical detection of OX-42 and hydroethidine staining were employed to determine the activation of microglia and production of reactive oxygen species (ROS), respectively. It was found that SST inhibited the LPS-induced microglial activity and ROS production. ELISA revealed a decreased production of pro-inflammatory mediators, including tumor necrosis factor- $\alpha$, interleukin-1 $\beta$ and prostaglandin E2 when SST was administered prior to LPS treatment. Western blot analysis showed that LPS-induced expression of inducible nitric oxide synthase, cyclooxygenase- 2 and nuclear factor $\kappa \mathrm{B}(\mathrm{NF}-\kappa \mathrm{B})$ p-p65 was attenuated by administration of SST prior to LPS application. The results indicated that LPS-induced loss of nigral DA neurons was inhibited by SST and the observed effects of SST on neuroprotection were associated with suppression of microglial activation and the NF- $\mathrm{KB}$ pathway, ensuing decreases of neuroinflammation and oxidative stress.
\end{abstract}

Correspondence to: Dr Yan Ren, Department of Neurology, The First Affiliated Hospital of China Medical University, 155 North Nanjing Street, Shenyang, Liaoning 110001, P.R. China E-mail: cmu1hrenyan@163.com

Key words: somatostatin, neuron, substantia nigra, microglia, Parkinson's disease
The present study therefore suggested that SST is beneficial for treating neurodegenerative diseases, such as PD, through inhibiting the activation of microglia.

\section{Introduction}

Parkinson's disease (PD) is a common neurodegenerative disease, leading to cell death of dopaminergic (DA) neurons in the substantia nigra (SN) (1). An approximate of 5-10\% of PD can be attributed to heritable genetic mutations; however, the cause of more common PD and the mechanisms underlying the development of the disease have remained elusive (2,3). Several lines of evidence suggest that activated microglia have a critical role in the pathogenesis of PD through producing inflammatory mediators, including tumor necrosis factor (TNF)- $\alpha$, interleukin (IL)-1 $\beta$ and neurotoxin, including inducible nitric oxide synthase (iNOS) and cyclooxygenase-2 (Cox-2) (4). Along with microglial activation, it has been reported that the production of reactive oxygen species (ROS) can accelerate the death of DA neurons (5). Increases of pro-inflammatory cytokines, activation of the nuclear factor $(\mathrm{NF})-\kappa \mathrm{B}$ signaling pathway and oxidative stress were observed in the destroyed DA neurons (6). Therefore, all these factors can be regarded as potential targets for treating PD.

Somatostatin (SST) as an inhibitor of growth hormone (GH) has been identified to be abundant throughout the central nervous system (7). As a neuromodulator, SST has a crucial role in memory and cognition (8). In addition, the decreased SST levels in the frontal and entorhinal cortex as well as the hippocampus have been correlated to the cognitive deficits in patients with PD (9). However, increases in SST have been observed in the cerebrospinal fluid of patients with early PD (10). Due to the controversial data reported, the function and mechanism of SST which participates in PD are still subject to extensive examination. Accumulating clinical and experimental evidence suggested that SST can provide potential therapy in neurodegenerative disorders involving cognitive dysfunctions (11). To date, little is known about the effects of SST in DA neurons in the context of PD and its mechanism of action. To investigate this matter, an animal model of PD was generated by injecting lipopolysaccharide (LPS) into the 
SN of rat brains. It was examined whether SST administered prior to LPS treatment was able to protect nigral DA neurons from LPS-induced neurotoxicity through inhibiting microglial activation and reducing subsequent neuroinflammation as well as oxidative stress. To clarify the functional mechanism of SST, the expression of NF- $\kappa \mathrm{B}$ p 65 and downstream factors were investigated in the present study.

\section{Materials and methods}

Reagents. The following reagents and kits were used in the present study: Rabbit polyclonal anti-tyrosine hydroxylase (TH; cat. no. 2792; Cell Signaling Technology, Inc., Boston, MA, USA), rabbit polyclonal anti-OX-42 (cat. no. orb11009; Biorbyt, Cambridge, UK), hydroethidine (Molecular Probes, Eugene, OR, USA), LPS (Sigma-Aldrich, St. Louis, MO, USA), heparin (Qianhong Bio-pharma Co., Ltd., Changzhou, China), SST (ProSpec, East Brunswick, NJ, USA), biotinylated goat anti-rabbit secondary antibody (cat. no. A0277) and horseradish peroxidase (HRP)-labeled streptavidin (Beyotime, Shanghai, China), bicinchoninic acid (BCA) kit (Beyotime), Rat TNF- $\alpha$ ELISA kit, Rat IL-1 $\beta$ ELISA kit and Prostaglandin E2 ELISA kit (PGE2; USCN, Wuhan, Hubei, China).

Stereotaxic surgery and SST administration. All experiments were performed according to the approved animal protocols and guidelines established by Chung et al (12). 144 female Sprague Dawley rats were provided by the Experimental Animal Centre of China Medical University (Shenyang, China). The animal study protocol was approved by the Animal Experimental Committee of China Medical University, and the mice received humane care according to the Principles of Laboratory Animal Care. They were randomly assigned to six experimental groups and received unilateral administration of $3 \mu \mathrm{l}$ phosphate-buffered saline (PBS), LPS (5 $\mu \mathrm{g}$ in $3 \mu \mathrm{l} \mathrm{PBS),} 2 \mu \mathrm{l}$ saline + LPS, $2 \mu \mathrm{l} \mathrm{SST}$ $(20 \mu \mathrm{g} / \mathrm{kg})+\mathrm{LPS}, 2 \mu \mathrm{l} \mathrm{SST}(40 \mu \mathrm{g} / \mathrm{kg})+\mathrm{LPS}, 2 \mu \mathrm{l} \mathrm{SST}$ (40 $\mu \mathrm{g} / \mathrm{kg})$ respectively, into the right $\mathrm{SN}$ as previously described by Chung et al (10). 24 rats of each treated group were separated into three different subgroups for various analyses, including OX-42 and hydroethidine tests of brains after $24 \mathrm{~h}$ of treatment, western blot and ELISA assays of $\mathrm{SN}$ after $24 \mathrm{~h}$ of treatment, as well as immunohistochemical detection of TH and Nissl in the SN after 7 days of treatment. SST was injected $1 \mathrm{~h}$ prior to LPS treatment.

Tissue preparation and immunohistochemistry. Brain tissues were prepared for immunohistochemical staining as previously reported (12). Tissues were dehydrated by using a graded ethanol series of $70 \%$ ethanol for $2 \mathrm{~h}, 80 \%$ overnight, $90 \%$ for $2 \mathrm{~h}$ and $100 \%$ for $2 \mathrm{~h}$. Brain tissues were then post-fixed in dimethylbenzene (China National Medicines Corporation Ltd., Beijing, China) for $30 \mathrm{~min}$ and embedded in dimethylbenzene-paraffin at $60^{\circ} \mathrm{C}$ for $2 \mathrm{~h}$, after which samples were embedded in a metal frame. Coronal sections $(5 \mu \mathrm{m})$ cut by a sliding microtome CM69001 (Leica Microsystems, Mannheim, Germany) were spread in warm water and placed onto glass slides to dry in a $70^{\circ} \mathrm{C}$-chamber for $40 \mathrm{~min}$. Sections were dewaxed using ethanol and then boiled in antigen retrieval solution for $10 \mathrm{~min}$. The cooled sections were incubated in $3 \% \mathrm{H}_{2} \mathrm{O}_{2}$ (China National Medicines Corporation Ltd.) for $15 \mathrm{~min}$ at room temperature and then blocked with normal goat serum (Beijing Solarbio Science \& Technology Co., Ltd., Beijing, China) for $15 \mathrm{~min}$. Sections were incubated overnight at room temperature with rabbit anti-OX-42 (1:100; cat. no. orb11009) for microglia and rabbit anti-TH (1:100; cat. no. 2792) for DA neurons. Following removal of unbound primary antibodies by washing, biotinylated goat anti-rabbit secondary antibody (1:200; cat. no. A0277) was added and incubated for $30 \mathrm{~min}$ at $37^{\circ} \mathrm{C}$. Sections were then washed with PBS and incubated with HRP-labeled streptavidin for $30 \mathrm{~min}$ at $37^{\circ} \mathrm{C}$. Finally, $100 \mu \mathrm{l}$ diaminobenzidine (Beijing Solarbio Science \& Technology Co., Ltd.) was added for coloration and hematoxylin stain (Beijing Solarbio Science \& Technology Co., Ltd.) for counterstaining. Hydroethidine (Molecular Probes) was used for in situ detection of $\mathrm{O}_{2}^{-}$and $\mathrm{O}_{2}{ }^{-}$-derived oxidants. For Nissl staining, a number of the $\mathrm{SN}$ tissue samples were stained in $0.5 \%$ cresyl violet (China National Medicines Corporation Ltd.). Following washing with water and dehydrating with ethanol as well as treating with dimethylbenzene (China National Medicines Corporation Ltd.), stained samples were analyzed under a stereo microscope (BX51; Olympus Corporation, Tokyo, Japan) or viewed with a confocal laser scanning microscope (FV1000S-SIM/IX81; Olympus Corporation).

Stereological estimation. The total number of TH-positive neurons was counted in the various groups at seven days post-injection (LPS, PBS, SST or a combination) using the stereo microscope BX51 (Olympus Corporation). This unbiased stereological method of cell counting according to a previously described method is not affected by either the counted elements (neurons) or the size of the reference volume (SN) (13).

Western blot analysis. For western blot analysis, protein was extracted from the $\mathrm{SN}$ of eight rats from each group following $24 \mathrm{~h}$ of treatment. Following determination of the protein concentration using a BCA kit, $40 \mu \mathrm{g}$ protein was boiled in PBS with $5 \mathrm{X}$ loading buffer (Beijing Solarbio Science \& Technology Co., Ltd.) for $5 \mathrm{~min}$. The bands of protein separated by SDS-PAGE ( $8 \%$ gel for iNOS, $10 \%$ gel for $N F-\kappa B$ p65 and NF- $\mathrm{NB}$ p-p65, and $12 \%$ gel for Cox-2) were transferred onto polyvinylidene difluoride membranes (Millipore, Billerica, MA, USA) using an electroblot apparatus (DYCZ-40D, Beijing, China). Filters were blocked in $5 \%$ non-fat milk and incubated separately overnight at $4{ }^{\circ} \mathrm{C}$ with the following primary antibodies: Rabbit polyclonal anti-iNOS (1:1,000; cat. no. bs-2072R; Bioss, Beijing, China), goat polyclonal anti-Cox-2 (1:100; sc-1747), mouse monoclonal anti-NF- $\mathrm{B}$ p-p65 (1:100; cat. no. sc-166748) and rabbit polyclonal anti-NF- $\mathrm{B}$ p65 (1:100; cat. no. sc-372) obtained from Santa Cruz Biotechnology, Inc. (Dallas, TX, USA). Membranes were then washed with Tris-buffered saline containing Tween-20 (TTBS) (BioSharp, Hefei, China) and incubated with the following corresponding HRP-conjugated secondary antibodies: Goat anti-mouse immunglobulin (Ig)G-HRP (1:5,000; cat. no. A0216), goat anti-rabbit IgG-HRP (1:5,000; cat. no. A0208) and donkey 

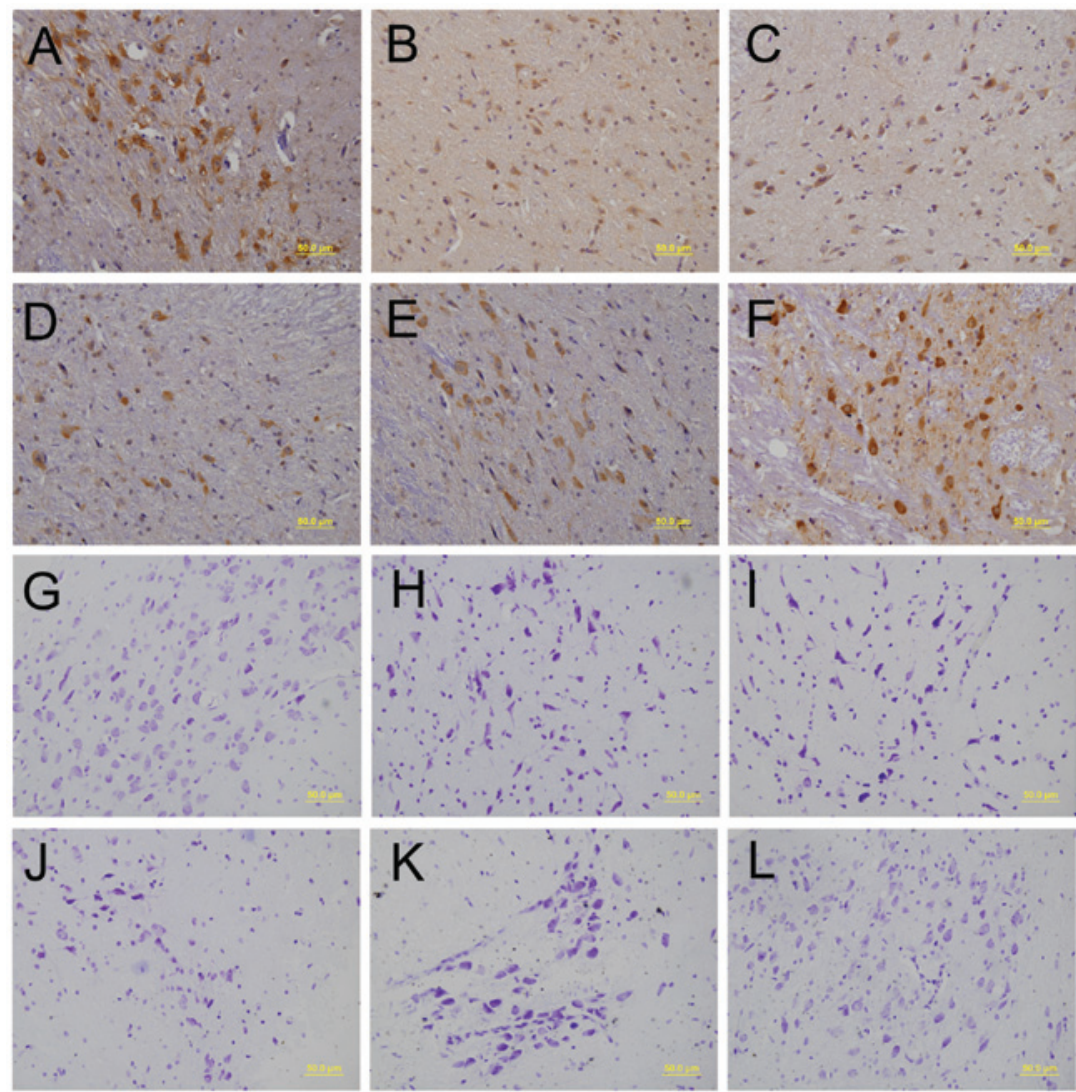

Figure 1. Effect of SST on LPS-induced loss of nigral dopaminergic neurons. Rats were sacrificed at the $7^{\text {th }}$ day after LPS injection, and their brains were selected for immunostaining with (A-F) tyrosine hyrdoxylase antibody and (G-L) Nissl staining. (A and G) Control treated with phosphate-buffered saline, (B and H) LPS model, (C and I) LPS + saline, (D and J) LPS + $20 \mathrm{~g} / \mathrm{kg} \mathrm{SST,} \mathrm{(E} \mathrm{and} \mathrm{K)} \mathrm{LPS}+40 \mu \mathrm{g} / \mathrm{kg} \mathrm{SST}$, and (F and L) $40 \mu \mathrm{g} / \mathrm{kg} \mathrm{SST}$. Scale bars, $50 \mu \mathrm{m}$. LPS, lipopolysaccharide; SST, somatostatin.

anti-goat IgG-HRP (1:5,000; cat. no. A0181) (Beyotime, Shanghai, China) for $45 \mathrm{~min}$ at $37^{\circ} \mathrm{C}$. Following washing with TTBS, protein bands were visualized using enhanced chemiluminescence reagent (cat. no. E002-5; Qihai Biotec, Shanghai, China). Protein levels were quantified by gray value analysis using Gel-Pro-Analyzer (Media Cybernetics, Inc., Rockville, MD, USA).

Measurement of TNF- $\alpha, I L-1 \beta$ and PGE2. The production of TNF- $\alpha$, IL- $1 \beta$ and PGE2 from the SN of rats was determined by ELISA. Proteins were extracted through homogenizing tissues and quantitated using the BCA kit. ELISA was then performed according to the manufacturer's instructions.

Statistical analysis. Values are expressed as the mean \pm standard deviation. All raw data were analyzed by one-way analysis of variance followed by the Bonferroni test for post hoc comparisons. Statistical analyses were performed using GraphPad Prism 5.0 software (GraphPad Software Inc., La Jolla, CA, USA). $\mathrm{P}<0.05$ was considered to indicate a statistically significant difference between values.

\section{Results}

Neuroprotective effect of SST on the LPS-treated SN. LPS injection into the SN was previously shown to result in a considerable loss of TH- and Nissl-positive cells, as well as
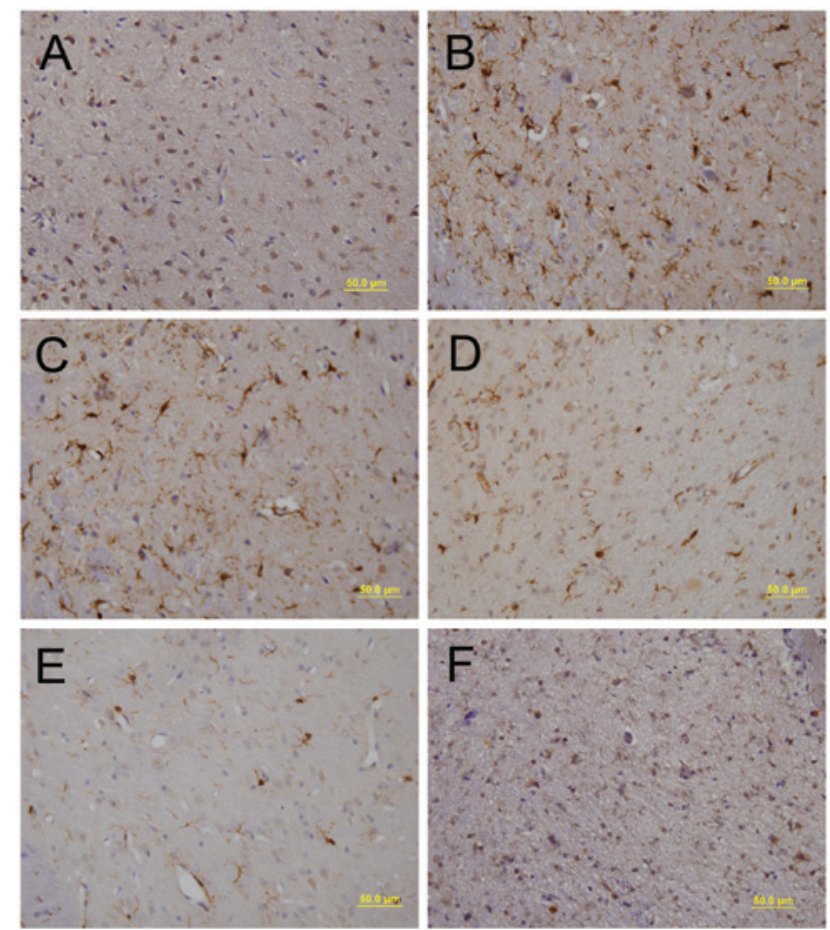

Figure 2. Effect of SST on LPS-induced microglial activation in the substantia nigra. Brains were immunostained with OX-42 antibody for analyzing the expression of OX-42. (A) Control treated with phosphate-buffered saline, (B) LPS model, (C) LPS + saline, (D) LPS + $20 \mu \mathrm{g} / \mathrm{kg}$ SST, (E) LPS + $40 \mu \mathrm{g} / \mathrm{kg} \mathrm{SST}$, (F) $40 \mu \mathrm{g} / \mathrm{kg}$ SST. Scale bars, $50 \mu \mathrm{m}$. LPS, lipopolysaccharide; SST, somatostatin. 


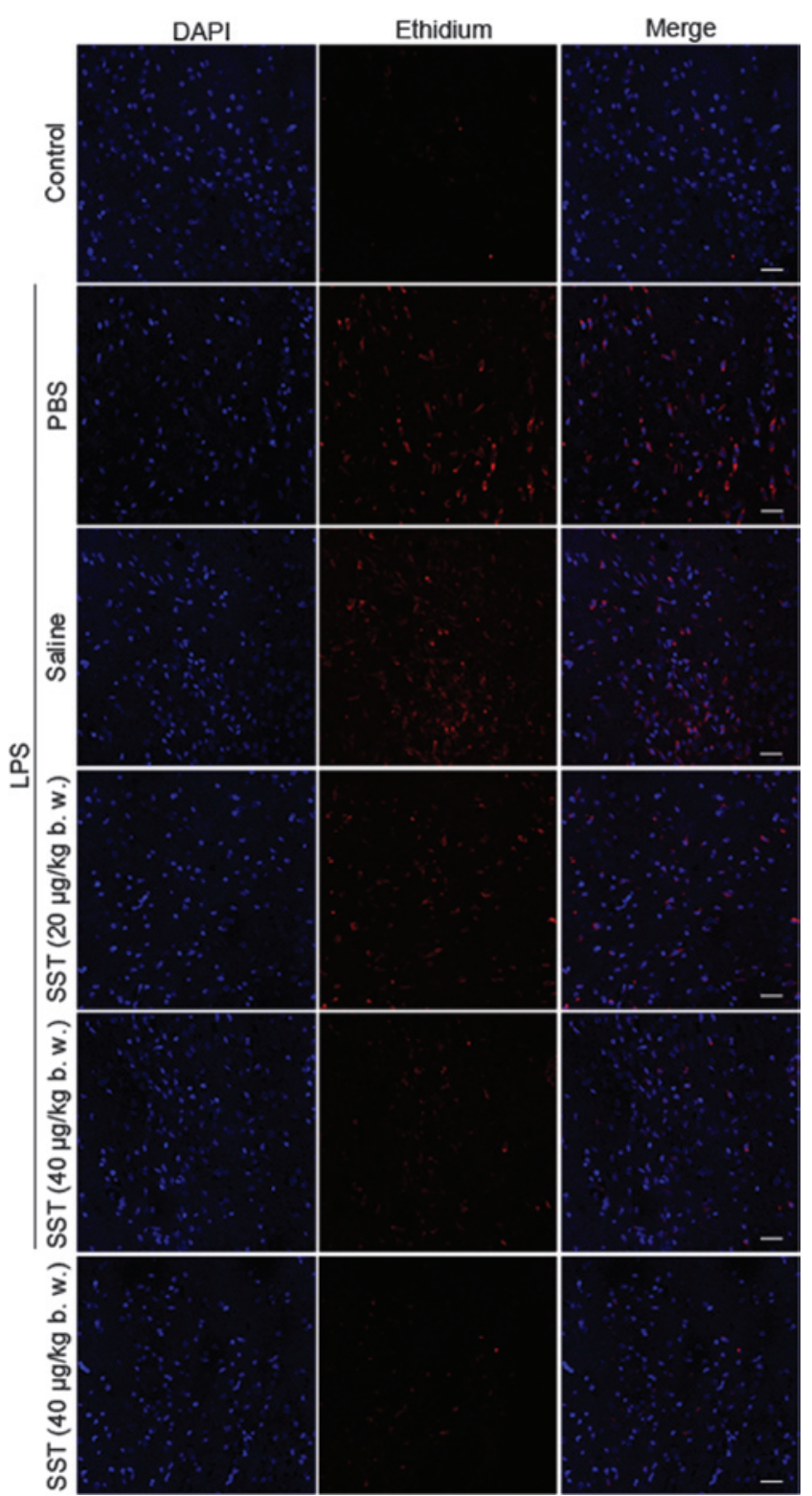

Figure 3. Effect of SST on LPS-induced production of reactive oxygen species. At $24 \mathrm{~h}$ following LPS injection in the presence or absence of SST, brains subjected to examination of the production of oxidized hydroethidine by testing the accumulated fluorescence through confocal laser scanning microscopy. Scale bars, $30 \mu \mathrm{m}$. LPS, lipopolysaccharide; SST, somatostatin; PBS, phosphate-buffered saline.

alterations in the morphology of TH-positive neurons to shrunken neuronal cell bodies $(12,13)$. To investigate the potential of SST to prevent LPS-induced neurotoxicity of nigral neurons, SST was administered $1 \mathrm{~h}$ prior to LPS injection. SST significantly attenuated the LPS-mediated loss of $\mathrm{TH}$-positive DA neurons in the $\mathrm{SN}$ and preserved normal neuronal morphology, as evidenced by an increased number of healthy cell bodies and processes with enhanced staining intensity (Fig. 1A-F). As shown in Fig. 1G, the morphology of the neurons were integrative and well-maintained. In Fig. $1 \mathrm{H}$, the number of healthy neurons was markedly decreased, and the number of dead neurons increased, as characterized by the chromatolysis of Nissl bodies and the presence of shrunken neuronal cell bodies with pyknotic nuclei surrounded by a thin band of cytoplasm. In Fig. 1K, the above pathological damages
A

B
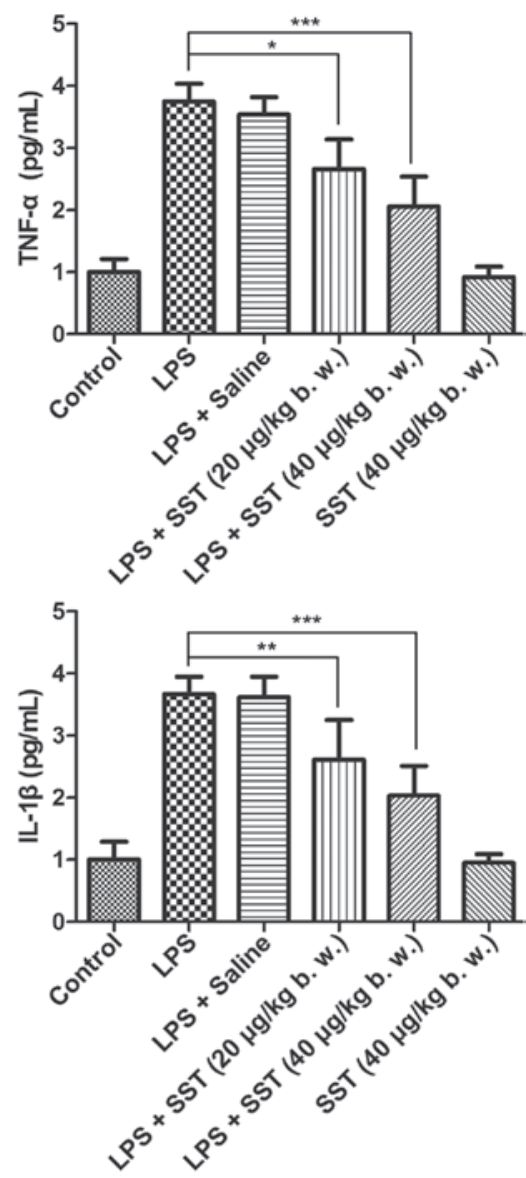

C

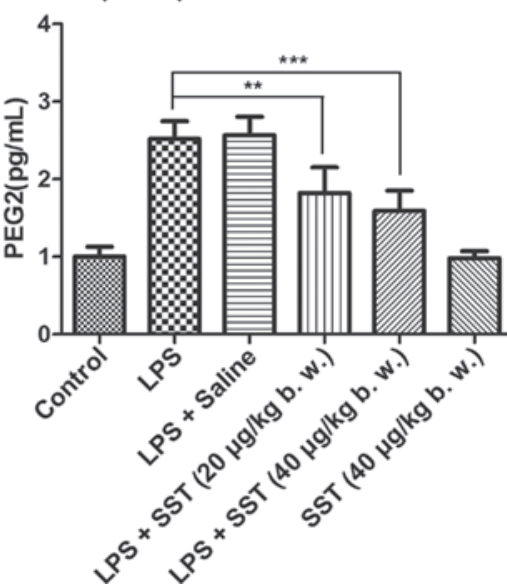

Figure 4. SST reduces the expression of LPS-induced pro-inflammatory mediators. At $24 \mathrm{~h}$ after LPS injection, the amounts of (A) TNF- $\alpha$, (B) IL- $1 \beta$ and (C) PGE2 in the SN were measured using ELISA. Values are presented as the mean \pm standard error of the mean of eight animals per group; ${ }^{*} \mathrm{P}<0.05$, ${ }^{* *} \mathrm{P}<0.01$ and ${ }^{* * * *} \mathrm{P}<0.001$ compared with the phosphate-buffered saline-injected SN. LPS, lipopolysaccharide; SST, somatostatin; SN, substantia nigra; TNF, tumor necrosis factor; IL, interleukin; PGE, prostaglandin E2.

were notably reduced, following pretreatment with higher concentrations of SST. The results of the LPS + saline group (Fig. 1I) were similar to those exhibited in Fig. 1H. In addition, the results of the LPS $+20 \mathrm{~g} / \mathrm{kg} \mathrm{SST}$ group (Fig. 1J) were similar, but not as obvious, as those exhibited in Fig. 1K, and the results of the $40 \mu \mathrm{g} / \mathrm{kg}$ SST group (Fig. 1L) were similar to the results shown in Fig. 1G, thus indicating that SST had no cytotoxic effects on the neurons. These results showed that SST had neuroprotective effects on LPS-treated SN. 

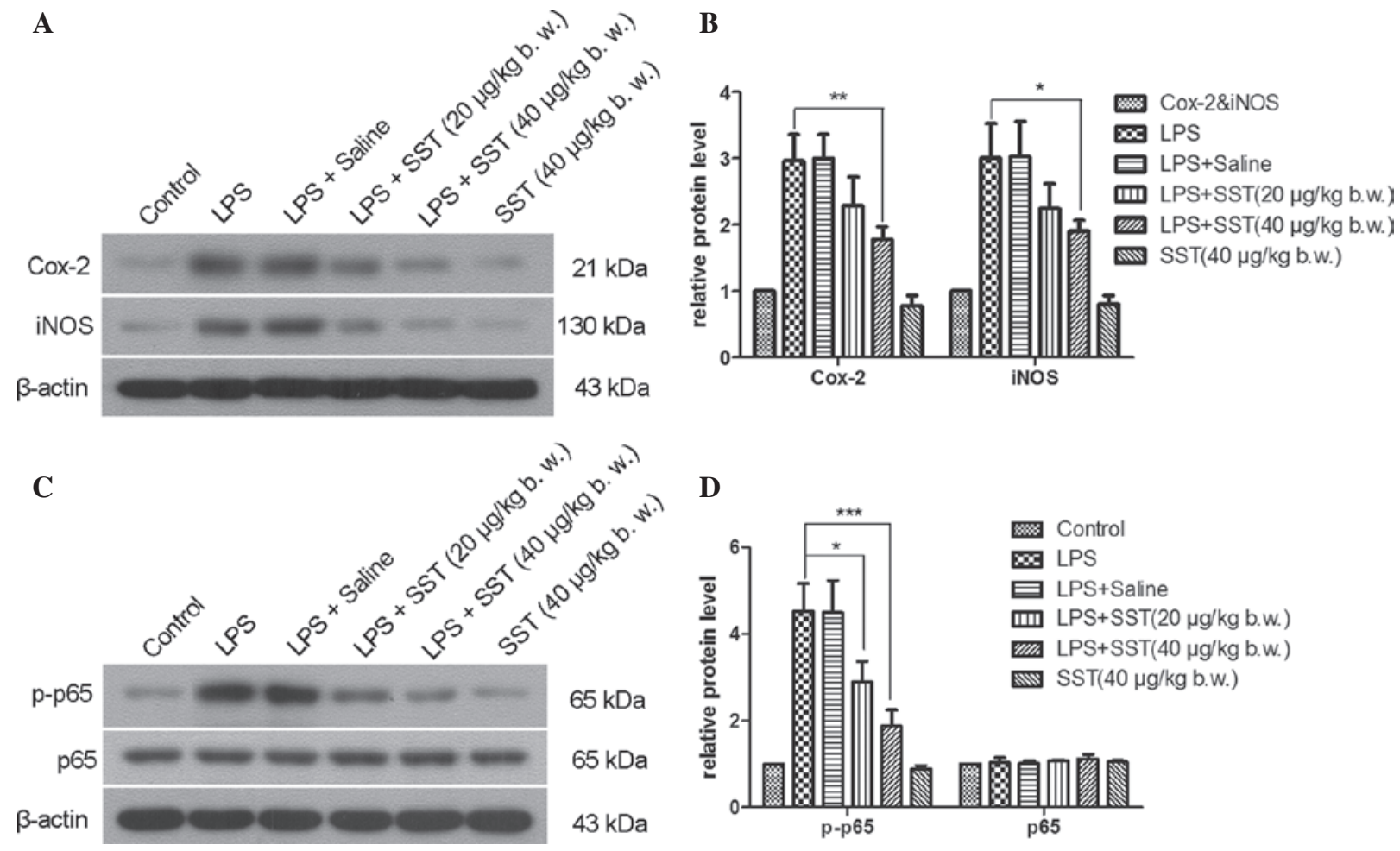

Figure 5. Effect of SST on LPS-induced expression of Cox-2, iNOS and the activation of NF- $\kappa$ B p65. 1. (A and C) representative western blot gels showing

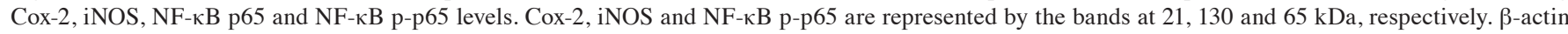
was used as an internal control. (B and D) Quantification of A and C by gray value analysis. Values are presented as the mean \pm standard error of the mean of eight animals per group. ${ }^{*} \mathrm{P}<0.05,{ }^{* *} \mathrm{P}<0.01,{ }^{* * * *} \mathrm{P}<0.001$ compared with phosphate-buffered saline-injected SN. LPS, lipopolysaccharide; SST, somatostatin; SN, substantia nigra; COX, cyclooxygenase; iNOS, inducible nitric oxide synthase; NF- $\kappa \mathrm{B}$, nuclear factor kappa B.

LPS-induced microglial activation is inhibited by SST. It has been reported that LPS is able to activate rat microglia and lead to neuronal death in vivo (14). Thus, the present study investigated the effect of SST on LPS-induced microglial activation in the SN. SN sections were prepared for immunohistochemical staining using antibodies against OX-42 to detect microglial activation. The majority of OX-42-positive microglia exhibited a resting morphology in the PBS-injected SN (Fig. 2A), whereas LPS-treated samples showed activated microglia with enhanced staining intensity and larger cell bodies with short, thick processes (Fig. 2B). Pre-treatment with SST dramatically decreased the number of activated microglia induced by LPS compared that in the saline-pre-treated control (Fig. 2C-E), while SST alone had no effect on microglial activation (Fig. 2F). These findings suggested that SST inhibited the LPS-induced activation of microglia.

LPS-induced ROS production is inhibited by SST. Recent studies have suggested that activated microglia are able to produce $\mathrm{O}_{2}{ }^{-}$and $\mathrm{O}_{2}{ }^{-}$-derived oxidants and they are thought to mediate the loss of nigral DA neurons in vitro and in vivo $(15,16)$. Hence, the present study investigated whether SST was able to enhance DA neuronal survival by inhibiting LPS-induced production of ROS. Accumulation of ethidium, the fluorescent product of oxidized hydroethidine, was significantly increased at $48 \mathrm{~h}$ in the LPS-treated SN compared with that in the PBS-injected controls, and the LPS-induced oxidant production was dramatically decreased by SST (Fig. 3). These results showed that SST inhibited LPS-induced ROS production.
SST decreases LPS-induced production of TNF- $\alpha, I L-1 \beta$ and $P G E 2$. Several studies have demonstrated that the production of TNF- $\alpha$, IL- $1 \beta$ and PGE2 are upregulated in LPS-injected SN $(14,17)$. Neuroinflammation is thought to mediate DA neuronal death in the SN (18). Therefore, the present study examined whether SST was able to decrease DA neuronal death by regulating LPS-induced production of TNF- $\alpha$, IL-1 $\beta$ and PGE2 in the SN. When $20 \mu \mathrm{g} / \mathrm{kg}$ SST was administered prior to LPS injection, the amount of TNF- $\alpha$, IL-1 $\beta$ and PGE2 was significantly reduced by $24.9 \%$ (Fig. 4A), 27.9\% (Fig. 4B) and $29.2 \%$ (Fig. $4 \mathrm{C}$ ), respectively. The production of TNF- $\alpha$, IL-1 $\beta$ and PGE2 was decidedly decreased by $41.8,43.9$ and $41.6 \%$, respectively, when $40 \mu \mathrm{g} / \mathrm{kg} \mathrm{SST}$ was administered prior to LPS treatment, while SST had no effect on the production of TNF- $\alpha$, IL-1 $\beta$ and PGE2 when it was used alone. This confirmed that SST was able to decrease LPS-induced production of TNF- $\alpha$, IL-1 $\beta$ and PGE2.

SST decreases LPS-induced overexpression of Cox-2, iNOS and $N F-\kappa B$ p-p65. Upregulation of Cox-2 and iNOS have been implicated in DA neuronal cell death of patients with degenerative brain diseases $(19,20)$. Inhibition of the activation of NF-kB p65 was able to prevent DA neuronal cell loss in a 1-methyl-4-phenyl-1,2,3,6-tetrahyropyridine-induced mouse model of PD (21). Accordingly, the present study examined the effect of SST on DA neuronal survival through affecting LPS-induced expression of Cox-2, iNOS and the activation of NF- $\mathrm{KB}$ in the SN. 24 hours following injection, LPS enhanced the expression of Cox-2, iNOS and activated NF- $\mathrm{kB}$ p 65 via phosphorylation (Fig. 5). Administration of $20 \mu \mathrm{g} / \mathrm{kg} \mathrm{SST}$ 
prior to LPS injection reduced the expression of Cox-2, iNOS and NF- $\kappa \mathrm{B}$ p-p65 by 23.4, 26.1 and $35.9 \%$, respectively. When $40 \mu \mathrm{g} / \mathrm{kg}$ SST was given prior to LPS injection, the expression of Cox-2, iNOS and NF- $\kappa$ B p-p65 was decreased by $40.5,37.3$ and $58.4 \%$, respectively. SST alone had no effects on Cox-2, iNOS and NF- $\mathrm{B}$ p-p65 expression. It was therefore demonstrated that SST was able to inhibit the expression of Cox-2, iNOS and NF-кB p-p65.

\section{Discussion}

PD is a complex neurodegenerative disorder owing to an aggravating process of neuronal loss within the SN (22). Microglial activation has been reported to induce the death of DA neurons (23). This is due to activated microglia being able to actively produce and secrete unfavorable toxic substances, including pro-inflammatory cytokines and ROS (24). The present study demonstrated that the neuroprotective effects of SST in the LPS-treated SN are based on the ability of SST to suppress microglial activity and thereby decrease the production of pro-inflammatory cytokines and ROS generation. Immunohistochemical staining of OX-42 suggested that SST inhibited microglial activation. Loss of nigral DA cells was decreased by administration of SST prior to LPS injection compared with LPS-treatment only, which was confirmed by immunohistochemical staining for TH and Nissl staining of the SN. Together with the results of a previous study (25), the present study proved that downregulation of microglial activity is able to improve survival of nigral DA neuronal cells. SST is therefore suggested as a potential drug for PD treatment.

Increasing evidence suggested that activated microglia are able to generate ROS, which results in oxidative stress to DA neurons in the $\mathrm{SN}$ of PD patients (26). $\mathrm{O}_{2}{ }^{-}$and $\mathrm{O}_{2}{ }^{-}$-derived oxidant molecules may exacerbate neurotoxicity (27). The accumulation of fluorescent oxidized hydroethidine showed that LPS-induced production of ROS may be mitigated through SST treatment prior to administration of LPS in the SN. Moreover, NO generated by iNOS also contributes to the oxidative stress associated with the neurotoxicity observed in PD (28). Importantly, neurodegeneration has been reported to be associated with up-regulation of iNOS expression in the SN (29). The present study showed that LPS-induced overexpression of iNOS was inhibited by treatment with SST. All these results suggested that LPS-induced activation of microglia and oxidative stress were prevented by SST, resulting in neuroprotection.

Neuroinflammation is an important feature in the progression of neurodegenerative disease (30). The activated microglia expresses pro-inflammatory cytokines including TNF- $\alpha$, IL-1 $\beta$ and PGE2, which lead to neuronal degeneration in the SN of PD patients (31). The results showed that LPS up regulated production of TNF- $\alpha$, IL-1 $\beta$ and PGE 2 was inhibited by SST application before LPS treatment in the SN. These findings are similar to previous reports that fucoidan significantly inhibits the release of TNF- $\alpha$ and prevents neurotoxicity in LPS-induced rat model of PD (32). It suggests that SST has the ability to inhibit the expression of pro-inflammatory cytokines, and lead to neuroprotection as an inhibitor of neuroinflammation.

In addition, a previous study showed that Cox-2 was rapidly upregulated when inflammation occurred, which appeared to be responsible for the formation of PGE2 and may have contributed to the neurodegenerative process in PD (33). Meanwhile, NF- $\kappa \mathrm{B}$ is thought to be a transcriptional controller, which participates in the release of Cox-2, following increased degeneration of DA neurons in an animal model of PD (34). The results of the present study showed that the expression of activated NF- $\kappa \mathrm{B}$ p65, Cox-2 and PGE2 in the SN was downregulated when SST was administered prior to LPS treatment compared with that in the group treated with LPS only. The NF- $\kappa \mathrm{B} / \mathrm{Cox}-2 / \mathrm{PGE} 2$ signaling pathway was confirmed to participate in the neurodegeneration of the LPS-induced PD model and the inflammatory responses of LPS-treated P12 cells proceed via the same mechanism (17). As suppression of $\mathrm{NF}-\kappa \mathrm{B}$ is able to attenuate the production of LPS-induced pro-inflammatory mediators (35), the inhibition of $\mathrm{NF}-\kappa \mathrm{B}$ may be a mechanism underlying the neuroprotective effect of SST. NF- $\kappa$ B, Cox-2 and PGE2 may therefore be considered as targets for PD treatment.

In conclusion, the findings of the present study demonstrated that SST, particularly at high concentrations of SST used in the SN, may inhibit ROS production, expression of pro-inflammatory cytokines and the $\mathrm{NF}-\kappa \mathrm{B}$ pathway as well as the activation of microglia, which may lead to increased neuronal survival. These results suggest that SST offers great therapeutic potential for treating neurodegenerative diseases, such as PD, through inhibiting microglial activation.

\section{Acknowledgements}

This study was supported by grants from the National Nature Science Foundation of China (no. 81371421) and the Social Development Project of Liaoning Province (no. 2011225020).

\section{References}

1. Ramos-Moreno T, Castillo CG and Martinez-Serrano A: Long term behavioral effects of functional dopaminergic neurons generated from human neural stem cells in the rat 6-OH-DA Parkinson's disease model. Effects of forced expression of BCL-X(L). Behav Brain Res 232: 225-232, 2012.

2. Golbe LI: Young-onset Parkinson's disease: A clinical review. Neurology 41: 168-173, 1991.

3. Zanon A, Rakovic A, Blankenburg $\mathrm{H}$, et al: Profiling of Parkin-binding partners using tandem affinity purification. PLoS One 8:e78648, 2013.

4. Choi SH, Joe EH, Kim SU and Jin BK: Thrombin-induced microglial activation produces degeneration of nigral dopaminergic neurons in vivo. J Neurosci 23: 5877-5886, 2003.

5. Song DY, Yu HN, Park CR, et al: Down-regulation of microglial activity attenuates axotomized nigral dopaminergic neuronal cell loss. BMC Neurosci 14: 112, 2013.

6. Yuan L, Wu Y, Ren X, Liu Q, Wang J and Liu X: Isoorientin attenuates lipopolysaccharide-induced pro-inflammatory responses through down-regulation of ROS-related MAPK/NF- $\kappa$ B signaling pathway in BV-2 microglia. Mol Cell Biochem 386: 153-165, 2014.

7. Epelbaum J, Guillou JL, Gastambide F, Hoyer D, Duron E and Viollet C: Somatostatin, Alzheimer's disease and cognition: An old story coming of age? Prog Neurobiol 89: 153-161, 2009.

8. Viollet C, Lepousez G, Loudes C, Videau C, Simon A and Epelbaum J: Somatostatinergic systems in brain: Networks and functions. Mol Cell Endocrinol 286: 75-87, 2008.

9. Epelbaum J, Ruberg M, Moyse E, Javoy-Agid F, Dubois B and Agid Y: Somatostatin and dementia in Parkinson's disease. Brain Res 278: 376-379, 1983.

10. Espino A, Calopa M, Ambrosio S, Ortolà J, Peres J and Navarro MA: CSF somatostatin increase in patients with early parkinsonian syndrome. J Neural Transm Park Dis Dement Sect 9: 189-196, 1995. 
11. Tuboly G and Vécsei L: Somatostatin and cognitive function in neurodegenerative disorders. Mini Rev Med Chem 13: 34-46, 2013.

12. Chung ES, Chung YC, Bok E, et al: Fluoxetine prevents LPS-induced degeneration of nigral dopaminergic neurons by inhibiting microglia-mediated oxidative stress. Brain Res 1363 : 143-150, 2010.

13. Chung ES, Bok E, Chung YC, Baik HH and Jin BK: Cannabinoids prevent lipopolysaccharide-induced neurodegeneration in the rat substantia nigra in vivo through inhibition of microglial activation and NADPH oxidase. Brain Res 1451: 110-116, 2012.

14. Liu M and Bing G: Lipopolysaccharide animal models for Parkinson's disease. Parkinson's Dis 2011: 327089, 2011.

15. Block ML, Zecca L and Hong JS: Microglia-mediated neurotoxicity: uncovering the molecular mechanisms. Nat Rev Neurosci 8: 57-69, 2007.

16. Koppula S, Kumar H, Kim IS and Choi D-K: Reactive oxygen species and inhibitors of inflammatory enzymes, NADPH oxidase, and iNOS in experimental models of Parkinson's disease. Mediators Inflamm 2012: 823902, 2012.

17. Geng Y, Fang M, Wang J, et al: Triptolide down-regulates COX-2 expression and PGE2 release by suppressing the activity of NF- $\kappa \mathrm{B}$ and MAP kinases in lipopolysaccharide-treated PC12 cells. Phytother Res 26: 337-343, 2012.

18. Tansey MG and Goldberg MS: Neuroinflammation in Parkinson's disease: Its role in neuronal death and implications for therapeutic intervention. Neurobiol Dis 37: 510-518, 2010.

19. Minghetti L: Role of COX-2 in inflammatory and degenerative brain diseases. In: Inflammation in the Pathogenesis of Chronic Diseases. Vol. 42. Harris R, Bittman R, Dasgupta D, et al (eds). Springer, Netherlands, pp127-141, 2007.

20. Broom L, Marinova-Mutafchieva L, Sadeghian M, Davis JB, Medhurst AD and Dexter DT: Neuroprotection by the selective iNOS inhibitor GW274150 in a model of Parkinson disease. Free Radic Biol Med 50: 633-640, 2011.

21. Aoki E, Yano R, Yokoyama H, Kato H and Araki T: Role of nuclear transcription factor kappa B (NF-kappaB) for MPTP (1-methyl-4-phenyl-1,2,3,6-tetrahyropyridine)-induced apoptosis in nigral neurons of mice. Exp Mol Pathol 86: 57-64, 2009.

22. Dexter DT and Jenner P: Parkinson disease: from pathology to molecular disease mechanisms. Free Radic Biol Med 62: 132-144, 2013.
23. Kim SR, Chung ES, Bok E, et al: Prothrombin kringle-2 induces death of mesencephalic dopaminergic neurons in vivo and in vitro via microglial activation. J Neurosci Res 88: 1537-1548, 2010.

24. Miller R, James-Kracke M, Sun G and Sun A: Oxidative and inflammatory pathways in Parkinson's disease. Neurochem Res 34: 55-65, 2009.

25. Shen W, Qi R, Zhang J, et al: Chlorogenic acid inhibits LPS-induced microglial activation and improves survival of dopaminergic neurons. Brain Res Bull 88: 487-494, 2012.

26. Park ES, Kim SR and Jin BK: Transient receptor potential vanilloid subtype 1 contributes to mesencephalic dopaminergic neuronal survival by inhibiting microglia-originated oxidative stress. Brain Res Bull 89: 92-96, 2012.

27. Lull M and Block ML: Microglial activation and chronic neurodegeneration. Neurotherapeutics 7: 354-365, 2010.

28. Hancock D, Martin ER, Vance JM and Scott WK: Nitric oxide synthase genes and their interactions with environmental factors in Parkinson's disease. Neurogenetics 9: 249-262, 2008.

29. Li M, Dai FR, Du XP, Yang QD and Chen Y: Neuroprotection by silencing iNOS expression in a 6-OHDA model of Parkinson's disease. J Mol Neurosci 48: 225-233, 2012.

30. Miller JA, Trout BR, Sullivan KA, Bialecki RA, Roberts RA and Tjalkens RB: Low-dose 1-methyl-4-phenyl-1,2,3,6-tetrahydropyridine causes inflammatory activation of astrocytes in nuclear factor- $\mathrm{\kappa B}$ reporter mice prior to loss of dopaminergic neurons. J Neurosci Res 89: 406-417, 2011.

31. More SV, Kumar H, Kim IS, Song SY and Choi DK: Cellular and molecular mediators of neuroinflammation in the pathogenesis of Parkinson's disease. Mediators Inflamm 2013: 952375, 2013.

32. Cui YQ, Jia YJ, Zhang T, Zhang QB and Wang XM: Fucoidan protects against lipopolysaccharide-induced rat neuronal damage and inhibits the production of proinflammatory mediators in primary microglia. CNS Neurosci Ther 18: 827-833, 2012.

33. Teismann P: COX-2 in the neurodegenerative process of Parkinson's disease. Biofactors 38: 395-397, 2012.

34. Phani S, Loike JD and Przedborski S: Neurodegeneration and inflammation in Parkinson's disease. Parkinsonism Relat Disord 18, (Suppl 1): S207-S209, 2012.

35. Kang CH, Jayasooriya RG, Choi YH, Moon SK, Kim WJ and Kim GY: $\beta$-Ionone attenuates LPS-induced pro-inflammatory mediators such as NO, PGE2 and TNF- $\alpha$ in BV2 microglial cells via suppression of the NF- $\mathrm{B}$ and MAPK pathway. Toxicol In Vitro 27: 782-787, 2013. 